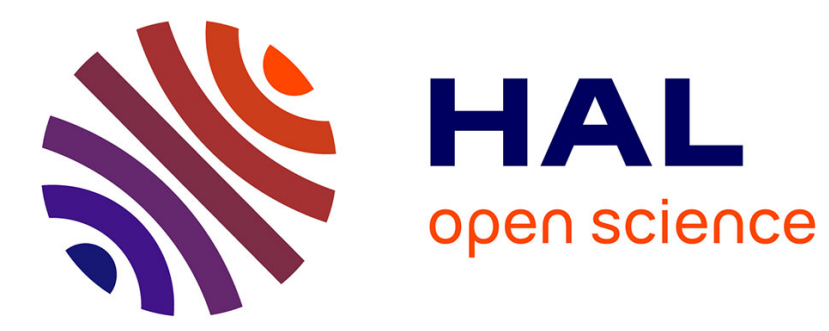

\title{
Antioxidants and glucose metabolism disorders
}

Catherine Bisbal, Karen Lambert, Antoine Avignon

\section{To cite this version:}

Catherine Bisbal, Karen Lambert, Antoine Avignon. Antioxidants and glucose metabolism disorders. Current Opinion in Clinical Nutrition and Metabolic Care, 2010, 13 (4), pp.439-446. 10.1097/MCO.0b013e32833a5559 . hal-02537079

\section{HAL Id: hal-02537079 \\ https://hal.umontpellier.fr/hal-02537079}

Submitted on 8 Apr 2020

HAL is a multi-disciplinary open access archive for the deposit and dissemination of scientific research documents, whether they are published or not. The documents may come from teaching and research institutions in France or abroad, or from public or private research centers.
L'archive ouverte pluridisciplinaire HAL, est destinée au dépôt et à la diffusion de documents scientifiques de niveau recherche, publiés ou non, émanant des établissements d'enseignement et de recherche français ou étrangers, des laboratoires publics ou privés. 


\section{Antioxidants and glucose metabolism disorders Catherine Bisbal $^{\mathrm{a}, \mathrm{b}}$, Karen Lambert ${ }^{\mathrm{a}, \mathrm{b}}$ and Antoine Avignon $^{\mathrm{a}, \mathrm{b}, \mathrm{c}}$}

aINSERM, ERI25, 'Muscle and Pathologies', 'Université Montpellier I, EA4202 and ${ }^{\mathrm{C}} \mathrm{CHU}$ Montpellier,

Montpellier, France

Correspondence to Antoine Avignon, Metabolic Disease Department, Lapeyronie Hospital, $371 \mathrm{Av}$

Doyen G. Giraud, 34295 Montpellier Cedex 5, France Tel: +33 4673384 06;

e-mail: a-avignon@chu-montpellier.fr

\section{Purpose of review}

Recent evidence suggests that oxidative stress is a cornerstone of the metabolic mechanisms by which overfeeding leads to insulin resistance. This review is an update of the most recent arguments in favor of this theory and of the possible role of antioxidants.

\section{Recent findings}

Reactive oxidative species (ROS) are produced by multiple pathways within the cell and are essential for many cellular functions. ROS production is balanced by enzymatic and nonenzymatic antioxidant systems. The perturbation of the pro-oxidant/antioxidant balance can lead to increased oxidative damage of macromolecules, a phenomenon known as 'oxidative stress'. ROS are involved both in insulin signal transduction and in insulin resistance when produced in excess. Overfeeding, saturated fatty acids, and obesity play a key role in the excessive production of ROS. However, a diet rich in fruits and vegetables, and therefore antioxidants, has demonstrated beneficial effects against oxidative damages and insulin resistance.

\section{Summary}

Experimental data are in favor of a beneficial role of antioxidants in glucose metabolism, but clinical data in humans are more controversial. Even if a diet rich in fruits and vegetables could provide an optimal mix of antioxidants, it remains unclear whether supplementation with antioxidants alone can reproduce the same effect.

\section{Keywords}

antioxidants, glucose metabolism, insulin resistance, oxidative stress

\section{Introduction}

Excess body weight and type 2 diabetes (T2D) are at the forefront of the growing epidemic of chronic diseases. They are at the core of a great number of cardiovascular disorders that still represent the leading cause of mortality in most developed countries. Apart from their impact on health and well being, these conditions have a considerable economic cost (http://www.cdc.gov/nchs/ FASTATS/lcod.htm). Unfortunately, the burden of obesity and its associated complications is not limited to economically developed countries and is increasingly affecting economically developing countries [1].

Diet and nutrition are key factors in the regulation of glucose metabolism. On the one hand, chronic overnutrition is associated with insulin resistance. On the other hand, nutrition itself is a tool in regulating glucose metabolism, as it has been shown that some types of foods like the Mediterranean diet might be protective [2]. The wealth of fruits and vegetables included in this diet, and consequently the supply of antioxidants, may be essential for its beneficial effects.
The objective of this review is to summarize recent evidence suggesting that oxidative stress is at the core of the mechanisms implicated in chronic overfeeding leading to insulin resistance and to consider the possible preventive role of antioxidants in these metabolic changes.

Reactive oxygen species and oxidative stress Reactive oxidative species (ROS), or free radicals, are atoms or molecules that have an unpaired electron that allows them to react with various molecules at the site of formation. They can contain both nitrogen and oxygen or only oxygen atoms (Table 1) and are produced by various physiological mechanisms. ROS are essential in several biochemical processes, including cellular differentiation, growth arrestment, apoptosis, immunity, and defense against microorganisms and intracellular messaging, including insulin signal transduction $\left[3^{\bullet \bullet}\right]$ (Figs 1 and 2).

Several target proteins of ROS, such as protein kinase $\mathrm{C}$ (PKC), play an important role in cellular metabolism and signal transduction [4]. For example, ROS are essential 


\section{Table 1 Principal reactive oxidative species}

\begin{tabular}{ll}
\hline Name & Formula \\
\hline Reactive oxygen species & \\
Singlet oxygen & \\
Superoxide radical & ${ }^{1} \mathrm{O}_{2}$ \\
Hydroxyl radical & $\mathrm{O}_{2}^{\bullet}$ \\
Hydrogen peroxide & ${ }^{\bullet} \mathrm{H}$ \\
Peroxide radical (lipid peroxide) & $\mathrm{H}_{2} \mathrm{O}_{2}$ \\
Alkoxyl radical & $\mathrm{ROO}^{\bullet}$ \\
Hypochlorous acid & $\mathrm{RO}^{\bullet}$ \\
Reactive nitrogen species & $\mathrm{HOCl}$ \\
Peroxynitrite radical & \\
Nitrogen dioxide radical & $\mathrm{ONOO}^{\bullet}$ \\
Alkyl peroxynitrite & $\mathrm{NO}_{2}^{\bullet}$ \\
\hline
\end{tabular}

regulators of insulin sensitivity and glucose homeostasis in vivo via tyrosine phosphorylation-dependent signaling and reversible inhibition of protein tyrosine phosphatases (PTPs) such as PTB1B and PTEN (phosphatase with tensin homology) $\left[5,6^{\circ}\right]$. According to the study by Loh et al. [6 $6^{\bullet}$, in-vivo production of $\mathrm{H}_{2} \mathrm{O}_{2}$, a process involving enhanced PTEN oxidation, also appears to be essential to maintain insulin sensitivity.

Therefore, ROS have a role in normal metabolism and 'oxidative stress' is an imbalance between the production of ROS and the defense systems against ROS (prooxidant-antioxidant balance). This imbalance can lead to a toxic state in which macromolecules (i.e., lipids, proteins, and/or DNA) are oxidatively damaged and cellular function is altered. Hence, although ROS are short-lived molecules, they leave detectable traces of modified oxidative products such as nitrated tyrosines and protein carbonyls as an indicator of protein oxidation. Isoprostanes, malondialdehyde, and 4-hydroxyl-2-nonenol are remnant signs of lipid peroxidation. In addition, the oxidized base, 8 -hydroxy-2'-deoxyguanosine $(8-\mathrm{OH}-$ $\mathrm{dG})$, is a marker of DNA oxidation.

The fight against free radicals involves the trapping of free radicals in nonenzymatic systems such as vitamin C,

Figure 1 Intracellular sources of reactive oxidative species

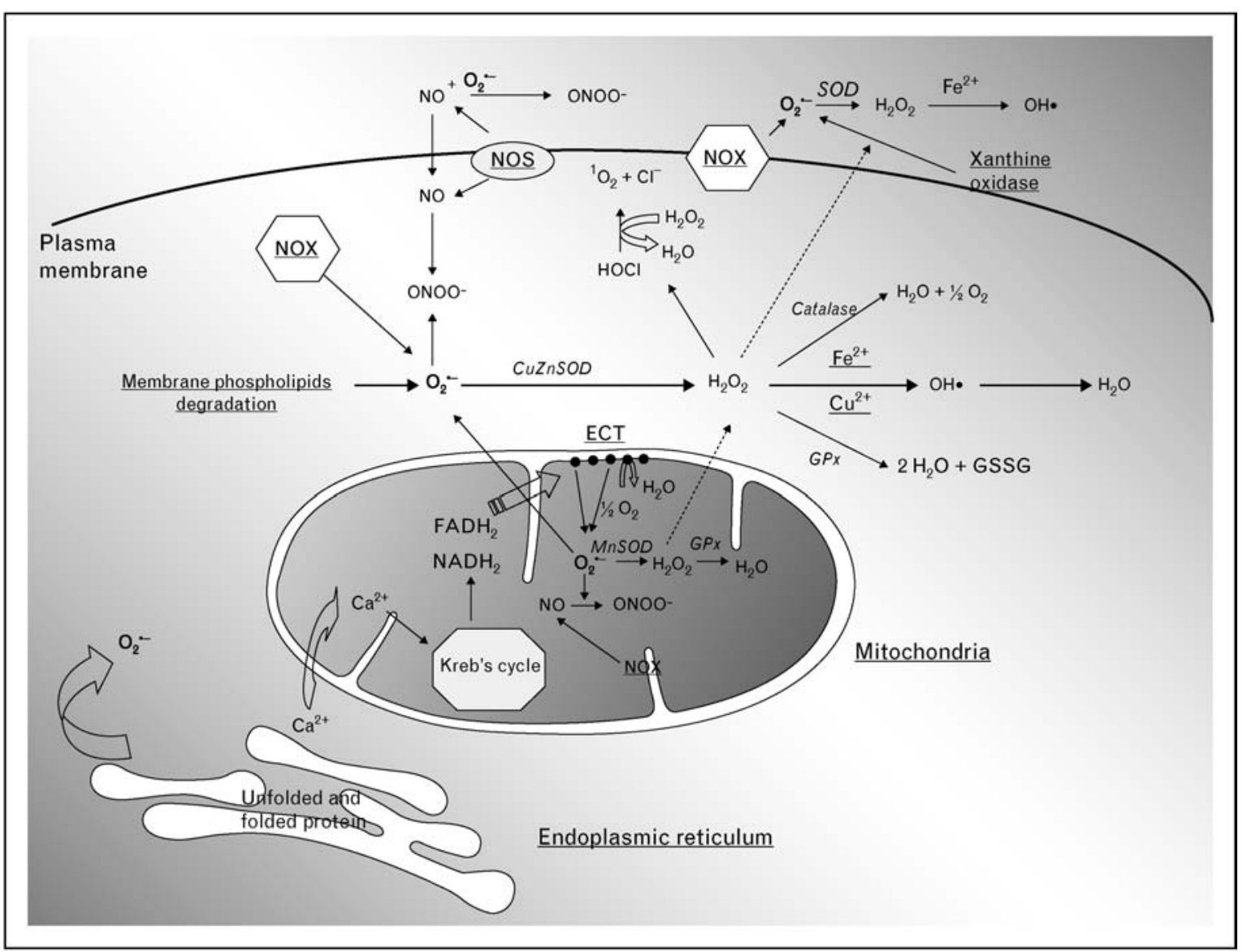

The main producers of reactive oxidative species (ROS) are the electron chain transporters (ECTs) NADPH oxidase (NOX), xanthine oxidase, and nitrite oxide synthase (NOS), which are underlined in this figure. The endoplasmic reticulum participates in ROS production during protein folding. There is also an exacerbation in ROS production during the unfolded protein response. Another potential source of ROS is the lipid membrane, particularly arachidonic acid degradation. The enzymatic antioxidant system is represented in bold and italics: superoxide dismutase (SOD) activated with manganese (MnSOD), copper, and zinc (CuZnSOD), catalase, glutathione peroxidase (GPx). 


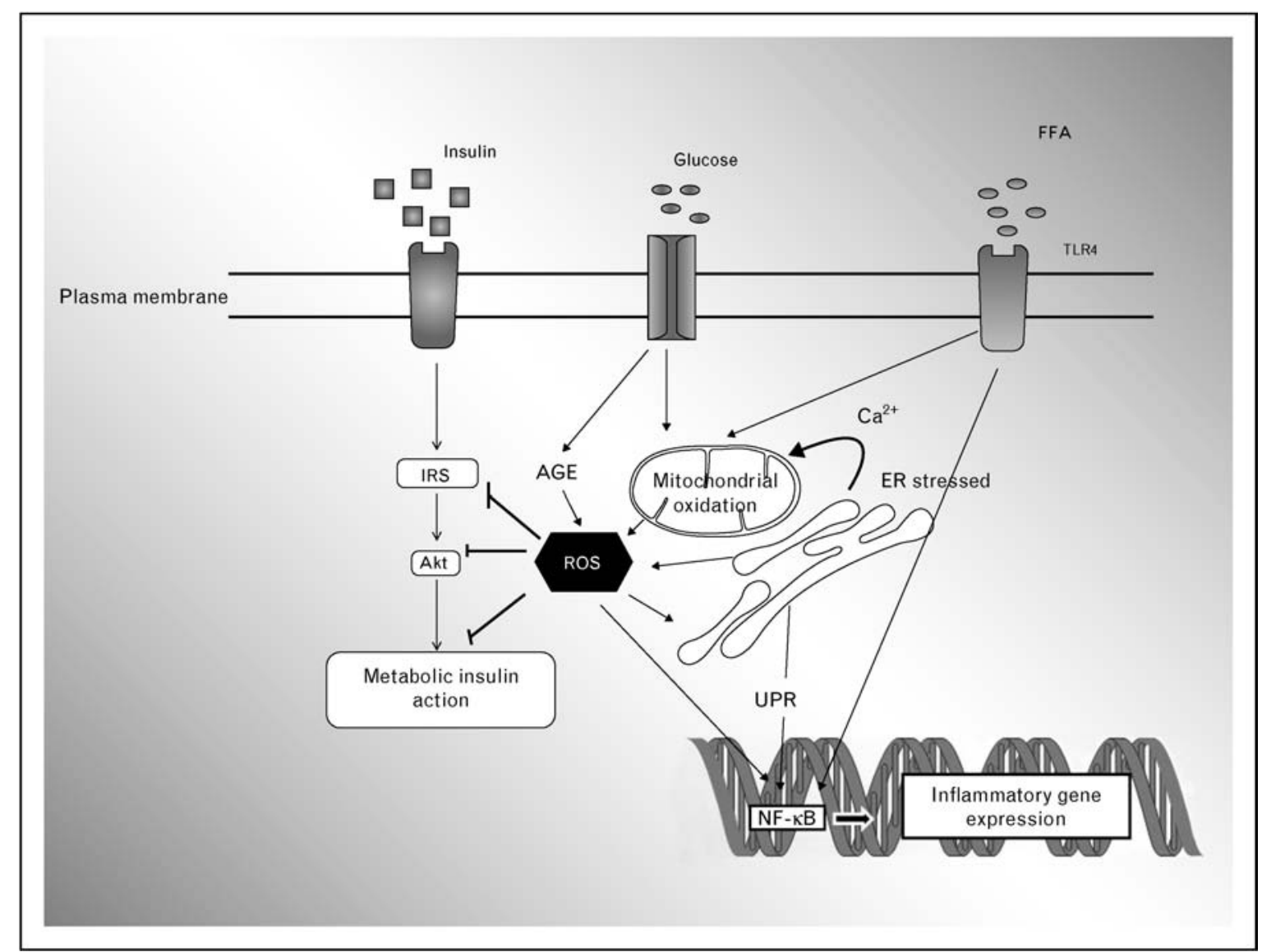

Reactive oxidative species (ROS) produced by one pathway (see Fig. 1) can inhibit insulin pathways at several levels. An increase in substrate availability [glucose and free fatty acids (FFAs)] can participate in ROS accumulation via advanced glycation end products (AGE) and mitochondrial alteration. Moreover, ROS and FFAs can modulate NF- $\mathrm{kB}$ activation, inducing inflammatory gene activation. Direct implications of inflammation cannot be ruled out, because FFAs can directly activate innate immunity via Toll-like receptor 4 (TLR4). ER, endoplasmic reticulum.

vitamin E, carotenoids, reduced glutathione, and polyphenols. It also involves enzymatic systems including, but not limited to, superoxide dismutases (SODs), catalase (CAT), and glutathione peroxidase (GPX). The antioxidant trace elements are cofactors of these enzymes: copper, zinc, and manganese for mitochondrial SOD and selenium for glutathione peroxidase [7].

\section{From oxidative stress to insulin resistance}

Insulin resistance is defined as a reduction in the action of insulin both in the cellular capture of glucose by muscle and fat cells and in the inhibition of hepatic glucose production. It is observed in more than $80 \%$ of obese patients and in almost all patients with a metabolic syndrome and/or T2D. In the case of T2D, insulin resistance appears several decades before the clinical manifestation of the disease and represents, alongside the insulin secretion defect, one of its two core pathophysiological mechanisms. The determinants of obesity- associated insulin resistance are both genetic and environmental; the environment plays an important role in the development of insulin resistance dependent upon a given genetic background. Despite scientific efforts, the initiating mechanisms of insulin resistance are only partially known, and oxidative stress represents only one hypothesis among many others $\left[3^{\bullet \bullet}\right]$.

Nevertheless, pathways leading to insulin resistance are multiple, including intracellular ceramide accumulation, activation of kinases such as c-Jun $\mathrm{N}$-terminal kinase (JNK), or inhibition of kinases such as kappa B kinase (IKK-b), mitogen-activated protein kinase (p38-MAPK) or protein kinase $\mathrm{C}(\mathrm{PKC})$ isoforms. In recent years, the role of inflammation and of endoplasmic reticulum stress has received increasing attention $\left[8^{\bullet \bullet}\right]$. In fact, the insulin signaling cascade constitutes a complex signaling network, each step being interconnected with others. The abrogation of one pathway can be compensated by another, thus allowing the propagation of the signal 
to the next step. Consequently, insulin resistance requires the alteration of several pathways before it becomes clinically evident.

In addition to obesity and metabolic syndromes, insulin resistance is encountered in situations as diverse as acute stress, pregnancy, hepatitis C, HIV protease inhibitor therapy, acromegaly and anti-inflammatory corticosteroid treatment. Thus, it appears logical to question whether one of the pathways could be an obligatory mechanistic common denominator linking these different forms of insulin resistance, an idea discussed by Houstis et al. [9]. In this study, they performed a genomic analysis of two very different cellular models of insulin resistance, one induced by treatment with the cytokine tumor-necrosis factor- $\alpha$ and the other with the glucocorticoid dexamethasone. Gene expression analysis suggested that ROS levels were increased in both models, which was confirmed through measures of the cellular redox state. Furthermore, different pharmacological and transgenic approaches that decrease ROS levels all ameliorated insulin resistance to varying degrees. One of these treatments was tested in obese insulin resistance mice and was shown to improve insulin sensitivity and glucose homeostasis. These results demonstrated that increased ROS levels are important triggers for creating insulin resistance in settings as different as inflammation and antiinflammatory treatment with corticosteroids. More recently, Hoehn et al. [10 ${ }^{\bullet \bullet}$ ] compared four different models of insulin resistance including chronic treatment with insulin, corticosteroids, proinflammatory cytokines, or lipids in both muscle and adipose cell lines. The study showed a direct correlation between mitochondrial oxidative stress and insulin resistance in all models. Once again, both pharmacologic and genetic strategies that override mitochondrial $\mathrm{O}_{2}{ }^{\bullet-}$ reversed or prevented the onset of insulin resistance both in vitro and in vivo. Moreover, selective induction of $\mathrm{O}_{2}{ }^{\bullet-}$ using the mitochondrial complex III antagonist, antimycin A, rapidly induced insulin resistance $\left[10^{\bullet \bullet}\right.$. Mitochondrial $\mathrm{O}_{2}{ }^{\bullet-}$ could, thus, be a unifying element of insulin resistance, acting principally as a nutrient sensor in key metabolic tissues to regulate nutrient intake in accord with the energy oversupply.

\section{From overnutrition to oxidative stress}

An association between obesity and oxidative stress that is improved after weight loss was highlighted in humans during the past decade and confirmed in recent studies $\left[11^{\bullet}-13^{\bullet}\right]$, some of which suggest that it could be mostly visceral fat that is responsible for this relationship [12 $\left.{ }^{\bullet}\right]$. The possible processes involved in obesity-associated oxidative stress are multiple and include the following: a high metabolic load that exposes cells to an overload of nutrients with excessive mitochondrial oxidation and enhanced ROS generation, inflammatory states associated with obesity, endoplasmic reticulum stress, and endocrine dysregulation such as high levels of insulin, which have been shown to be associated with increased ROS production (Figs. 1 and 2). For more details on this subject, the reader may refer to the review by Bashan $e t a l$. $\left[3^{\bullet \bullet}\right]$. Interestingly, regardless of weight gain, increased fat intake is capable of shifting the cellular redox environment to a more oxidized state in the skeletal muscle of both rodents and humans, as shown by Anderson et al. $\left[14^{\bullet \bullet}\right]$. Mitochondrial production of hydrogen peroxide in the skeletal muscle of lean volunteers is doubled within $4 \mathrm{~h}$ after a high-fat meal (65\% fat), a phenomenon also observed after some 5 days of a high-fat diet. These effects are also observed in rodents in association with insulin resistance and are prevented by pharmacological and genetic strategies to control mitochondrial oxidative stress. Hence, the oversupply of substrates has a direct effect on muscle cell function and involves an imbalance in the redox environment, with the mitochondria playing a central role. Other studies show that NADPH oxidase is also involved in the increased production of ROS in response to saturated fatty acids in muscle cells $\left[15^{\circ}\right]$. Therefore, it is the metabolic burden of fat intake that appears to be responsible for oxidative stress and insulin resistance, regardless of whether obesity is present. In addition, saturated fatty acids regulate chemotactic factors expressed in cultured adipose cells by a mechanism that involves ROS generation and innate immunity via a Toll-like receptor 4 (TLR4)-dependent pathway [16 $\left.{ }^{\bullet \bullet}\right]$. If such data were to be confirmed in vivo in humans, they would highlight the importance of dietary fat and oxidative stress in macrophage accumulation in adipose tissue, a hallmark of obesity implicated in systemic inflammation and insulin resistance.

The role of fatty acids cannot be confined to the obese population. Some people have a low expandability of their adipose tissue, meaning their adipocytes have a reduced capacity to accommodate an increase of fat storage. Even in the absence of excess body weight, these individuals are particularly sensitive to dietary fat $\left[17^{\circ}\right]$. The inability of adipocytes to expand sufficiently to store lipids in excess leads to cellular hypertrophy; the oxygen supply of these hypertrophic adipocytes is reduced, inducing hypoxia and, in turn, an increase in inflammatory cytokine production, macrophage accumulation, lipolysis and apoptosis $\left[17^{\bullet}, 18^{\bullet}\right]$.

Finally, the finding in animal experiments that the boost in ROS production precedes both the increase in inflammatory markers and the onset of insulin resistance favors a causal role of oxidative stress in these disorders $\left[10^{\bullet \bullet}, 19^{\bullet}\right]$. Therefore, many arguments demonstrate that oxidative stress plays a central role in the initiating mechanisms of peripheral insulin resistance. High-fat 
feeding and chronic fatty acid exposure are also associated with increased ROS production, both in the $\beta$-cells, leading to dysfunction and cell death [20 $0^{\circ}$, and in the liver, leading to hepatic insulin resistance $\left[21^{\bullet}\right]$. Thus, oxidative stress induced by overnutrition could be involved at all levels of the pathophysiological mechanisms of T2D.

\section{Dietary antioxidants and glucose metabolism}

In a number of the works we have mentioned so far, we have seen that the use of pharmacological and transgenic antioxidant approaches in cell culture or animals was able to reverse or prevent insulin resistance induced by fatty acids or overfeeding $\left[9,10^{\bullet \bullet}, 14^{\bullet \bullet}, 15^{\bullet}\right]$. Many experimental and animal studies show that dietary antioxidants, taken either as extracts or as a part of the food itself, also have beneficial effects on glucose metabolism. An incomplete list of the antioxidants with the most recent references supporting their effect on glucose metabolism is presented in Table $2\left[22,23^{\bullet}, 24-28,29^{\bullet}, 30,31^{\bullet}, 32,33\right.$, $\left.34^{\bullet \bullet}, 35^{\bullet \bullet}, 36,37,38^{\bullet}, 39^{\bullet \bullet}, 40^{\bullet \bullet}\right]$. However, it is important to note that their benefits are not necessarily found at all levels of glucose metabolism. For example, dietary quercetin was found to have no effect on hepatic insulin resistance of mice subjected to a high-fat diet [ $\left[41^{\circ}\right]$, whereas it decreased inflammatory markers in parallel with a transient increase in energy expenditure [ $\left.42^{\circ}\right]$.

However, interventional studies that have directly assessed the effects of antioxidants on glucose metabolism in humans are rare. Very few have used the insulin clamp, the gold standard method used to evaluate insulin sensitivity. Only the effects of supplementation with alpha lipoic acid (ALA), or with vitamins $\mathrm{C}$ and $\mathrm{E}$ alone or in association, on insulin sensitivity have been studied using this method, and each has shown positive effects $[33,37,43]$. Nevertheless, the published evidence is ambiguous, as apparent in the recent study by Ristow

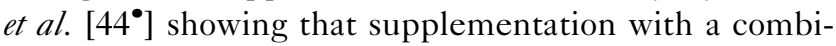
nation of vitamins $\mathrm{C}$ and $\mathrm{E}$ at high doses may preclude the health-promoting effect of exercise on improved insulin sensitivity. However, high levels of vitamin C, associated with a healthy diet rich in fruits and vegetables, have a preventive effect on T2D [38 ${ }^{\circ}$ and do not reduce the positive effect of exercise $\left[45^{\circ}\right]$. Regarding the polyphenols, only one study has evaluated the effects of cocoa consumption on insulin sensitivity using the insulin clamp in essential hypertensive patients and did not detect any effect [46].

If antioxidants are beneficial to glucose metabolism, it seems logical to expect a preventive effect on the occurrence of T2D, which has been confirmed by almost all epidemiological studies. A meta-analysis of previously published studies confirms the protective effect of vitamin $\mathrm{E}$ and carotene consumption on the occurrence of T2D [22], an effect that could be mediated by an improvement in insulin sensitivity, as recently confirmed both by the Insulin Resistance and Atherosclerosis (ARIC) Study $\left[34^{\bullet \bullet}\right]$ and the Uppsala Longitudinal Study of Adult Men (ULSAM) [35*0]. Nevertheless, all larger intervention trials evaluating the diabetes-preventive potential of antioxidant supplements have been unable to find any positive effects of supplementation, with the most recently published study being the Women's Antioxidant Cardiovascular Study (WACS) and the SU.VI.MAX study (SUpplementation en VItamines et

Table 2 Main dietary source of antioxidant compounds with demonstrated activities on glucose metabolism, type 2 diabetes, or metabolic syndrome

\begin{tabular}{|c|c|}
\hline Antioxidant compound $\left[22,23^{\bullet}\right]$ & Main dietary sources $[24,25]$ \\
\hline \multicolumn{2}{|l|}{ Polyphenols } \\
\hline \multicolumn{2}{|l|}{ Flavonoids [26-28] } \\
\hline Flavonols: quercetin, myricetin, kampeferol, rutin & $\begin{array}{l}\text { Peas, carrot, broccoli, spinach, cauliflower, apple, plum, apricot, } \\
\text { strawberries, aloe vera (leaves), tomatoes, black and green tea, } \\
\text { asparagus }\end{array}$ \\
\hline Flavanols: catechins, epicatechin & Cocoa, black chocolate, black and green tea \\
\hline Anthocyanins: cyaniding, delphinidin, luteolinidin & $\begin{array}{l}\text { Blackberries, raspberries, blueberries, orange, eggplant, cherries, } \\
\text { red grape, red wine, purple corn }\end{array}$ \\
\hline Isoflavones: genistin, formononetin, coumestrol & Soy, blackbeans, red clover, alfalfa, peanut \\
\hline Flavanones: hesperidin, naringenin & Orange, grapefruit, lemon, lime, tomato (skin) \\
\hline Flavones: apigenin, luteolin, tangeritin & Parsley, celery, sweet pepper \\
\hline \multicolumn{2}{|l|}{ Phenolic acids $\left[23^{\circ}, 29^{\circ}\right]$} \\
\hline Hydroxybenzoic acid derivatives: gallic acid, ellagic acid & Black and green tea, red wine, berries, potatoes \\
\hline $\begin{array}{l}\text { Hydroxycinnamic acid derivatives: chlorogenic acid, caffeic } \\
\text { acid, hydrocinnamic acid }\end{array}$ & $\begin{array}{l}\text { Blueberries, coffee, kiwi fruit, apples, pears, red wine, broccoli, } \\
\text { plums, cherries }\end{array}$ \\
\hline \multicolumn{2}{|l|}{ Stilbenoids } \\
\hline Transresveratrol $\left[30,31^{\bullet}, 32\right]$ & Skin of red grapes, cranberries, blueberries, bilberries \\
\hline Vitamin E: tocopherol $\left[33,34^{\bullet \bullet}, 35^{\bullet \bullet}, 36\right]$ & $\begin{array}{l}\text { Unheated vegetable oil: wheat germ oil, palm oil, cereals, almonds, } \\
\text { hazelnuts, green vegetables (spinach), butter, milk, egg, avocado, } \\
\text { oily fish (tuna) }\end{array}$ \\
\hline Vitamin C: L-ascorbic acid $\left[37,38^{\circ}\right]$ & Kakadu, camu camu, acerola, jujube, broccoli, Brussels sprout, lychee \\
\hline Lipoic acid $\left[39^{\bullet \bullet}\right]$ & Spinach, broccoli \\
\hline Carotenoids: $\beta$-cryptoxanthin, $\beta$-carotene, lutein $\left[35^{\bullet \bullet}, 40^{\bullet \bullet}\right]$ & Alfalfa, carrot, tomato, grapefruit, watermelon \\
\hline
\end{tabular}


Minéraux AntioXydants) $\left[47^{\bullet \bullet}, 48^{\bullet \bullet}\right]$. Moreover, in a systematic review analyzing the effects of $\beta$-carotene, vitamins $\mathrm{A}$ and $\mathrm{E}$, vitamin $\mathrm{C}$, and selenium, on all-cause mortality in adults included in primary and secondary prevention trials, Bjelakovic et al. [49] did not find convincing evidence that these antioxidants supplementations had beneficial effects on diabetes prevention. In fact, supplementation with $\beta$-carotene, vitamin $A$, or vitamin $\mathrm{E}$ seemed to increase the risk of death. Data on phenolic compounds are much rarer and provide mixed results. The analysis of data from the 38000 women of the Women's Health Study (WHS) does not show any link between flavonoid consumption and diabetes risk [26,27], but tea and coffee consumption is associated with a diabetes-protective effect that could be related to their polyphenol content $\left[23^{\bullet}, 29^{\circ}\right]$.

\section{Antioxidants: beyond the antioxidant effect}

Although we see that the experimental data are overwhelmingly in favor of a beneficial role of antioxidants on glucose metabolism, clinical data in humans are less convincing. Several explanations can be given. As previously mentioned, excess ROS can negatively interfere with insulin signal transduction, but their presence in small quantities is ultimately essential for insulin action $\left[3^{\bullet \bullet}\right.$. An excess of antioxidants may prove deleterious, which might help explain the results of some studies. Second, antioxidants, when they have reacted with ROS, become pro-oxidants and must interact with other antioxidants. A chain reaction involving different antioxidants must be put in place until a product is achieved that does not have enough reducing power to interact with the macromolecular compounds in the cell [50]. Thus, perhaps a diet rich in fruits and vegetables could provide an optimal mix of antioxidants, but it is unclear whether supplementation with some antioxidants alone can reproduce the same effects. In addition, it cannot be excluded that the beneficial effects of fruits and vegetables on the prevention of diabetes may be due to other bioactive compounds than the antioxidants themselves.

Finally, the effects of most molecules are not solely confined to their antioxidant properties, which may ultimately prove to be of secondary importance. One of the most striking examples is resveratrol, a phenolic compound of the stilbene family that has antioxidant properties and is capable of interacting directly with numerous metabolic pathways $\left[31^{\bullet}\right]$. At high doses, resveratrol improves the insulin sensitivity of mice fed a high-fat diet [30], prevents diet-induced obesity and alleviates obesity-related insulin resistance [32]. These effects are most likely mediated via SIRT1, a protein deacetylase that plays a role in the extension of lifespan and in chromatin remodeling. Its mechanism of action is associated with gene silencing rather than direct antioxidant action [51]. Similarly, vitamin E may not be confined to its antioxidant effects, it participates directly in controlling the expression of certain genes [36].

\section{Conclusion}

Experimental studies in cell cultures and animals have shown that ROS may play a central role in the mechanisms linking excess food and saturated fat intake and altered glucose metabolism. Epidemiologic studies have shown that a diet rich in fruits and vegetables has beneficial effects on insulin sensitivity and diabetes prevention, which has been attributed to their antioxidant properties. However, human studies hardly support a beneficial effect of antioxidants supplementation on glucose metabolism, suggesting that specific combinations of antioxidants may be necessary to observe these effects. It is also possible that the health benefits of such diets might not be solely attributed to their antioxidant capacity but could involve synergistic mechanisms between the different molecules. Further studies are needed to clarify the potential role of antioxidants in the improvement or prevention of abnormal glucose metabolism.

\section{References and recommended reading}

1 Kelly T, Yang W, Chen CS, et al. Global burden of obesity in 2005 and projections to 2030. Int J Obes (Lond) 2008; 32:1431-1437.

2 Giugliano D, Esposito K. Mediterranean diet and metabolic diseases. Curr Opin Lipidol 2008; 19:63-68.

3 Bashan N, Kovsan J, Kachko I, et al. Positive and negative regulation of insulin -• signaling by reactive oxygen and nitrogen species. Physiol Rev 2009; 89:2771.

This is an essential review for all those who are interested in the relationship between ROS and insulin action. It enables the reader to have a clear understanding of the cellular origin of ROS and of their involvement, on one hand, in insulin signal transduction and, on the other hand, in insulin resistance. In fact, it must be kept in mind that these reactive species are not only implicated in development of insulin resistance but that they are also essential signal transducers in insulin pathways.

4 Knapp LT, Klann E. Superoxide-induced stimulation of protein kinase C via thiol modification and modulation of zinc content. J Biol Chem 2000; 275:24136-24145.

5 Elchebly M, Payette P, Michaliszyn E, et al. Increased insulin sensitivity and obesity resistance in mice lacking the protein tyrosine phosphatase-1B gene. Science 1999; 283:1544-1548.

6 Loh K, Deng H, Fukushima A, et al. Reactive oxygen species enhance insulin - $\quad$ sensitivity. Cell Metab 2009; 10:260-272.

In this study, the authors demonstrate that in-vivo production of $\mathrm{H}_{2} \mathrm{O}_{2}$ is essential to insulin action. The absence of glutathione peroxidase 1 in mice (Gpx1-/L mice), a key enzyme in the elimination of physiological ROS, protects from highfat-diet-induced insulin resistance in relation with enhanced oxidation of PTEN.

7 Fang YZ, Yang S, Wu G. Free radicals, antioxidants, and nutrition. Nutrition 2002; 18:872-879.

8 Hotamisligil GS, Erbay E. Nutrient sensing and inflammation in metabolic -• diseases. Nat Rev Immunol 2008; 8:923-934.

In this important review, the authors present an integration of metabolism and immunity showing their convergence at many levels. These pathways are coordinated to maintain homeostasis. Dysfunction in one of them could lead to metabolic diseases. 
9 Houstis N, Rosen ED, Lander ES. Reactive oxygen species have a causal role in multiple forms of insulin resistance. Nature 2006; 440:944-948.

10 Hoehn KL, Salmon AB, Hohnen-Behrens C, et al. Insulin resistance is a - cellular antioxidant defense mechanism. Proc Natl Acad Sci U S A 2009; 106:17787-17792.

An important article showing that oxidative stress is a common feature of different models of insulin resistance and that mitochondrial $\mathrm{O}_{2}{ }^{\bullet-}$ production precedes insulin resistance development.

11 Krzystek-Korpacka M, Patryn E, Boehm D, et al. Advanced oxidation protein

- $\quad$ products (AOPPs) in juvenile overweight and obesity prior to and following weight reduction. Clin Biochem 2008; 41:943-949.

This study highlights the importance of oxidative stress in juvenile obesity and the effect of different diets, exercise, and weight reduction in decreasing oxidative stress.

12 Gletsu-Miller N, Hansen JM, Jones DP, et al. Loss of total and visceral adipose

- tissue mass predicts decreases in oxidative stress after weight-loss surgery. Obesity (Silver Spring) 2009; 17:439-446.

This study does also highlight the relationship between obesity, weight loss, and oxidative stress. It shows a specific association between adiposity and lipid peroxides, a measure of oxidative damage.

13 Brown LA, Kerr CJ, Whiting P, et al. Oxidant stress in healthy normal-weight, - overweight, and obese individuals. Obesity (Silver Spring) 2009; 17:460466.

This study complements the previous two references, showing the importance of recent work demonstrating a relationship between obesity and oxidative stress.

14 Anderson EJ, Lustig ME, Boyle KE, et al. Mitochondrial $\mathrm{H}_{2} \mathrm{O}_{2}$ emission and

-. cellular redox state link excess fat intake to insulin resistance in both rodents and humans. J Clin Invest 2009; pii:37048.

This is a major publication. The authors establish that mitochondrial $\mathrm{H}_{2} \mathrm{O}_{2}$ is a critical factor in the cause of high-fat-diet-induced insulin resistance, with $\mathrm{H}_{2} \mathrm{O}_{2}$ serving as a gauge of energy balance and a regulator of redox status within myofibers. The production of $\mathrm{H}_{2} \mathrm{O}_{2}$ increases very quickly after taking a single highfat meal in humans.

15 Lambertucci RH, Hirabara SM, Silveira Ldos R, et al. Palmitate increases

- superoxide production through mitochondrial electron transport chain and NADPH oxidase activity in skeletal muscle cells. J Cell Physiol 2008; 216:796-804.

The authors have tested the main sites of ROS production in skeletal muscle after palmitic acid incubation and eliminated xanthine oxidase in ROS generation.

16 Han CY, Kargi AY, Omer M, et al. Differential effect of saturated and

-. unsaturated free fatty acids on the generation of monocyte adhesion and chemotactic factors by adipocytes: dissociation of adipocyte hypertrophy from inflammation. Diabetes 2009; 59:386-396.

This study is important because it shows the direct effect of fatty acids on the outbreak of inflammatory response of adipocytes and on monocytes adhesion. It also helps to differentiate the effects of various fatty acids at this level and provides arguments for locating ROS upstream of these actions.

17 Lionetti L, Mollica MP, Lombardi A, et al. From chronic overnutrition to insulin

- resistance: the role of fat-storing capacity and inflammation. Nutr Metab Cardiovasc Dis 2009; 19:146-152.

This review describes how a chronically positive energy balance may lead to insulin resistance if the adipose tissue expandability is low and places endoplasmic reticulum stress as well as adipose tissue inflammation as primary alterations. This review contains an interesting integrative figure.

18 Gutierrez DA, Puglisi MJ, Hasty AH. Impact of increased adipose tissue mass

- on inflammation, insulin resistance, and dyslipidemia. Curr Diab Rep 2009; 9:26-32.

This review highlights the role of adipose tissue in insulin resistance and T2D development.

19 Matsuzawa-Nagata $\mathrm{N}$, Takamura $\mathrm{T}$, Ando $\mathrm{H}$, et al. Increased oxidative stress

- precedes the onset of high-fat diet-induced insulin resistance and obesity. Metabolism 2008; 57:1071-1077.

This study shows an upregulation of genes implicated in ROS generation in liver and adipose tissue of mice fed a high-fat diet before inflammation and insulin resistance. The limitation of this work is the discrepancy between mRNA data and the consequences of oxidative stress in liver.

20 Andrikopoulos S. Obesity and type 2 diabetes: slow down! Can metabolic - deceleration protect the islet beta cell from excess nutrient-induced damage? Mol Cell Endocrinol 2010; 316:140-146.

Although the present review focuses on peripheral insulin resistance, it is important not to overlook that in situation of overfeeding and obesity, all body cells will be subjected to nutrients overload. This is particularly the case of pancreatic cells. Hence, Andrikopoulos postulates that a reduction in the ability to metabolize excess fat may actually protect the function and viability of beta cell by reducing endoplasmic reticulum stress and ROS production.

21 Raffaella C, Francesca B, Italia F, et al. Alterations in hepatic mitochondrial

- compartment in a model of obesity and insulin resistance. Obesity (Silver Spring) $2008 ; 16: 958-964$.

This study emphasizes the relationship between high-fat feeding, obesity, oxidative stress, and insulin resistance at the hepatic level.
22 Hamer M, Chida Y. Intake of fruit, vegetables, and antioxidants and risk of type 2 diabetes: systematic review and meta-analysis. J Hypertens 2007; $25: 2361-2369$

23 Huxley R, Lee CM, Barzi F, et al. Coffee, decaffeinated coffee, and tea

- consumption in relation to incident type 2 diabetes mellitus: a systematic review with meta-analysis. Arch Intern Med 2009; 169:2053-2063.

With this meta-analysis of over 500000 individuals with over 21000 new cases of T2D, the authors confirm an inverse association between coffee, decaffeinated coffee, and tea consumption and subsequent risk of diabetes.

24 D'Archivio M, Filesi C, Di Benedetto R, et al. Polyphenols, dietary sources and bioavailability. Ann Ist Super Sanita 2007; 43:348-361.

25 Ostertag LM, O'Kennedy N, Kroon PA, et al. Impact of dietary polyphenols on human platelet function: a critical review of controlled dietary intervention studies. Mol Nutr Food Res 2010; 54:60-81.

26 Nettleton JA, Harnack $\sqcup$, Scrafford CG, et al. Dietary flavonoids and flavonoid-rich foods are not associated with risk of type 2 diabetes in postmenopausal women. J Nutr 2006; 136:3039-3045.

27 Song Y, Manson JE, Buring JE, et al. Associations of dietary flavonoids with risk of type 2 diabetes, and markers of insulin resistance and systemic inflammation in women: a prospective study and cross-sectional analysis. J Am Coll Nutr 2005; 24:376-384.

28 Karthick M, Stanely Mainzen Prince P. Preventive effect of rutin, a bioflavonoid, on lipid peroxides and antioxidants in isoproterenol-induced myocardial infarction in rats. J Pharm Pharmacol 2006; 58:701-707.

29 van Dieren S, Uiterwaal CS, van der Schouw YT, et al. Coffee and tea - consumption and risk of type 2 diabetes. Diabetologia 2009; 52:25612569.

As the precedent analysis, this study of a cohort of 38176 men and women shows that drinking coffee or tea is associated with a lowered risk of T2D, which cannot be explained by magnesium, potassium, caffeine or blood pressure effects.

30 Baur JA, Pearson KJ, Price NL, et al. Resveratrol improves health and survival of mice on a high-calorie diet. Nature 2006; 444:337-342.

31 Harikumar KB, Aggarwal BB. Resveratrol: a multitargeted agent for age- associated chronic diseases. Cell Cycle 2008; 7:1020-1035.

This important review provides an update on the many targets of resveratrol as described in the literature. Numerous studies showed that resveratrol possess anticancer, antidiabetic and antiaging properties. These targets have potential therapeutic interest.

32 Lagouge M, Argmann C, Gerhart-Hines Z, et al. Resveratrol improves mitochondrial function and protects against metabolic disease by activating SIRT1 and PGC-1alpha. Cell 2006; 127:1109-1122.

33 Paolisso G, D'Amore A, Giugliano D, et al. Pharmacologic doses of vitamin E improve insulin action in healthy subjects and noninsulin-dependent diabetic patients. Am J Clin Nutr 1993; 57:650-656.

34 Costacou T, Ma B, King IB, Mayer-Davis EJ. Plasma and dietary vitamin E in -. relation to insulin secretion and sensitivity. Diabetes Obes Metab 2008; 10:223-228.

Plasma $\alpha$-tocopherol concentrations are known to exert a protective effect on diabetes incidence among persons not taking vitamin $E$ supplements. To understand at what level $\alpha$-tocopherol act (insulin sensitivity, insulin secretion or both), the authors analyzed $\alpha$-tocopherol, insulin sensitivity, and insulin secretion from 457 patients during a 5 -year follow-up study. They show that plasma concentration of $\alpha$-tocopherol is associated with insulin sensitivity.

35 Arnlov J, Zethelius B, Riserus U, et al. Serum and dietary beta-carotene and -• alpha-tocopherol and incidence of type 2 diabetes mellitus in a communitybased study of Swedish men: report from the Uppsala Longitudinal Study of Adult Men (ULSAM) study. Diabetologia 2009; 52:97-105.

A 27 -year follow-up study of 846 men aged 50 years showing that impaired antioxidant status (lower concentrations of circulating and dietary $\beta$-carotene and $\alpha$-tocopherol) independently predicts the development of insulin resistance and T2D.

36 Valastyan S, Thakur V, Johnson A, et al. Novel transcriptional activities of vitamin E: inhibition of cholesterol biosynthesis. Biochemistry 2008; 47:744752.

37 Paolisso G, D'Amore A, Balbi V, et al. Plasma vitamin C affects glucose homeostasis in healthy subjects and in noninsulin-dependent diabetics. Am J Physiol 1994; 266:E261-E268.

38 Harding $\mathrm{AH}$, Wareham NJ, Bingham SA, et al. Plasma vitamin $\mathrm{C}$ level, fruit and

- vegetable consumption, and the risk of new-onset type 2 diabetes mellitus: the European prospective investigation of cancer: Norfolk prospective study. Arch Intern Med 2008; 168:1493-1499.

The authors of this study have found a strong inverse association between diabetes risk and plasma vitamin $\mathrm{C}$ level and to a lesser degree with fruit and vegetable intake. 
39 Poh Z, Goh KP. A current update on the use of alpha lipoic Acid in the -. management of type 2 diabetes mellitus. Endocr Metab Immune Disord Drug Targets 2009; 9:392-398.

This review describes the beneficial effect of ALA on the management of T2D and some of its complications. ALA is not only a free radical scavenger and a potent antioxidant but also a regulator of metabolic pathway.

40 Sluijs I, Beulens JW, Grobbee DE, van der Schouw YT. Dietary carotenoid

-. intake is associated with lower prevalence of metabolic syndrome in middleaged and elderly men. J Nutr 2009; 139:987-992.

This cross-sectional study deals with the dietary intake of carotenoids on metabolic syndrome and associated risk factor. The main result is a beneficial effect of carotenoid intake on these parameters with a substantial main effect due to $\beta$-carotene and lycopene.

41 Stewart LK, Wang Z, Ribnicky D, et al. Failure of dietary quercetin to alter the

- temporal progression of insulin resistance among tissues of C57BL/6J mice during the development of diet-induced obesity. Diabetologia 2009; 52:514523.

In this study, the authors show that initiation and development of insulin resistance induced by high-fat diet is not uniform across tissues, with impairment primarily in the liver that is not ameliorated by dietary quercetin.

42 Stewart LK, Soileau JL, Ribnicky D, et al. Quercetin transiently increases - energy expenditure but persistently decreases circulating markers of inflammation in C57BL/6J mice fed a high-fat diet. Metabolism 2008; 57:S39-46.

Unlike the previous reference in which quercetin, a flavonoid, had no effect on hepatic insulin resistance, the authors show here that it increases energy expenditure while reducing inflammation; these results suggest a potential effect of quercetin in the chronic low-level inflammatory state associated with obesity.

43 Jacob S, Henriksen EJ, Schiemann AL, et al. Enhancement of glucose disposal in patients with type 2 diabetes by alpha-lipoic acid. Arzneimittelforschung 1995; 45:872-874.

44 Ristow M, Zarse K, Oberbach A, et al. Antioxidants prevent health-promoting

- effects of physical exercise in humans. Proc Natl Acad Sci U S A 2009; 106:8665-8670.

In this study, the authors show that exercise-induced ROS formation is essential to insulin sensitivity in humans and that this beneficial effect of exercise on metabolism is almost completely abrogated by daily ingestion of the commonly used antioxidants vitamin $\mathrm{C}$ and vitamin $\mathrm{E}$ in high doses.
45 Thamer C, Machicao F, Stefan N, et al. High baseline vitamin C levels do not - $\quad$ prevent a positive outcome of a lifestyle intervention. Diabetes Care 2009; 32:e112.

This study emphasizes the importance of vitamin $C$ intake through a diet rich in fruits and vegetables on glucose metabolism. Importantly, high vitamin levels did not prevent the beneficial effect of life intervention.

46 Muniyappa R, Hall G, Kolodziej TL, et al. Cocoa consumption for 2 wk enhances insulin-mediated vasodilatation without improving blood pressure or insulin resistance in essential hypertension. Am J Clin Nutr 2008; 88:1685-1696.

47 Czernichow S, Vergnaud AC, Galan P, et al. Effects of long-term antioxidant

- supplementation and association of serum antioxidant concentrations with risk of metabolic syndrome in adults. Am J Clin Nutr 2009; 90:329335.

This prospective study also demonstrates the lack of beneficial effect of antioxidant combination supplementation on the risk of metabolic syndrome development. However, baseline $\beta$-carotene and vitamin $C$ levels were negatively associated with the risk of metabolic syndrome underlining the current recommendations to consume antioxidant-rich foods.

48 Song $Y$, Cook NR, Albert CM, et al. Effects of vitamins $C$ and $E$ and

-. beta-carotene on the risk of type 2 diabetes in women at high risk of cardiovascular disease: a randomized controlled trial. Am J Clin Nutr 2009; 90:429-437.

This prospective study found no effect of vitamin supplementation on the risk of developing diabetes even though there was a trend toward a modest reduction with vitamin $\mathrm{C}$ and toward a slight increase with vitamin $\mathrm{E}$.

49 Bjelakovic G, Nikolova D, Gluud LL, et al. Mortality in randomized trials of antioxidant supplements for primary and secondary prevention: systematic review and meta-analysis. JAMA 2007; 297:842-857.

50 Blomhoff R. Dietary antioxidants and cardiovascular disease. Curr Opin Lipidol 2005; 16:47-54.

51 Sun C, Zhang F, Ge X, et al. SIRT1 improves insulin sensitivity under insulinresistant conditions by repressing PTP1B. Cell Metab 2007; 6:307319. 